\title{
A CLINICAL STUDY ON ASSOCIATION OF METABOLIC SYNDROME AND CARDIOVASCULAR RISK IN PSORIASIS
}

\author{
Venna Ashok Babu1, K. Sri Venkateswaran², R. M. Rajasekar ${ }^{3}$
}

${ }^{1}$ Associate Professor, Department of Dermatology (DVL), Mamatha General Hospital, Khammam, Telangana. 2 Professor and HOD, Department of DVL, Vinayaka Mission Medical College and Hospital, Karaikal, Pondicherry. ${ }^{3}$ Consultant Dermatologist, Thanjavur.

\section{ABSTRACT}

\section{BACKGROUND}

Psoriasis is one of the most common skin condition in dermatology. The association of metabolic syndrome is increasing in psoriatic patients. To educate and treat the patients for the associated conditions is equally important.

Aim is to estimate and co-relate the association of obesity, lipid profiles, blood pressure and blood sugars (metabolic syndrome), and evaluate the cardiovascular risk in psoriatic patients.

\section{MATERIALS AND METHODS}

A clinical study of 50 conformed cases of psoriasis who attended the outpatient department of dermatology were studied. ECG, lipid profiles and relevant investigations were done. At the same time, 20 healthy individuals (controls) were also studied. Leven's test for equality of variances and t-test for equality of means are used. P value of $<0.05$ was significant.

\section{RESULTS}

Out of Hypertension, Diabetes, Obesity and Hyperlipidaemia any two were positive in one case (2.79\%), any three were positive in 14 cases (38.88\%), and all four were positive in 21 cases (58.33\%). Among control, 1 was suffering from metabolic syndrome. When compared to control, the P value was significant for body mass index (BMI), blood sugars, hypertension.

\section{CONCLUSION}

Psoriasis is a definite risk for metabolic syndrome and cardiovascular morbidity. So early diagnosis and treatment for metabolic syndrome is equally important along with treatment of psoriasis.

\section{KEYWORDS}

Metabolic Syndrome, Cardiovascular Risk, Psoriasis.

HOW TO CITE THIS ARTICLE: Babu VA, Venkateswaran KS, Rajasekar RM. A clinical study on association of metabolic syndrome and cardiovascular risk in psoriasis. J. Evolution Med. Dent. Sci. 2016;5(93):6859-6868, DOI: 10.14260/Jemds/2016/1551

\section{BACKGROUND}

Psoriasis is one of the most common conditions in dermatology outpatient departments, the prevalence being $0.8 \%-5.6 \%$. Among psoriasis patients, there is increased incidence of hypertension, diabetes, obesity and hyperlipidaemias in the recent past. There are two clinical presentations of psoriasis. Type 1 disease (hereditary form) associated with HLACw6, this accounts for more than 75\% of the cases, a positive family history, onset before 40 years, more severe and recurrent. Type 2 disease (sporadic) which starts later in life and without any family history or HLACw6 association. ${ }^{1}$ Psoriasis occurs with almost equal frequency in males and females. But high prevalence in males has been noted in Indian studies. ${ }^{2}$

Psoriasis is a $\mathrm{T}$ cell mediated autoimmune chronic inflammatory disease. ${ }^{3}$ There appears to be a strong relationship between psoriasis and fat metabolism. Studies

Financial or Other, Competing Interest: None.

Submission 14-10-2016, Peer Review 08-11-2016,

Acceptance 14-11-2016, Published 21-11-2016.

Corresponding Author:

Dr. Venna Ashok Babu,

H. No. 11-2-80/1, Balaji Nagar,

Wyra Road,

Khammam-507001.

E-mail: drashok24@gmail.com

DOI: $10.14260 /$ jemds $/ 2016 / 1551$ indicate raised levels of serum triglycerides, serum cholesterol, low density lipoproteins (LDL), very low density lipoproteins(VLDL) and decreased high density lipoproteins (HDL). It has also been observed that lipid abnormalities could precede the onset of psoriasis and may be genetically determined.4-6

The higher proportion of patients with psoriasis have been observed to have hypertension. ${ }^{7}$ This association may have a common basis. Angiotensin II, product of angiotensin converting enzyme is known to regulate vascular tone and to stimulate the release of pro-inflammatory cytokines that are involved in the pathogenesis of psoriasis. ${ }^{8}$ Likewise a rise in plasma rennin activity has been observed in psoriasis. ${ }^{9}$

An association between psoriasis and raised fasting blood glucose levels, hyperinsulinaemia, insulin resistance and type 2 diabetes has been observed.7 Insulin resistance was attributed to inflammation, and elevated C-reactive protein levels are predictive of diabetes. Long term use of topical steroids on large body surface areas could explain the observed increase in risk for diabetes in psoriasis. Reports indicate that acute phase reactants, such as C-reactive protein are significantly raised in psoriasis patients as compared to healthy controls, even during the remission phase. ${ }^{10,11}$ It is possible that the increased risk of cardiovascular disease in psoriasis may be due to these persistently raised reactants. ${ }^{12}$

Among all the possible comorbidities, excess adipose tissue and macrophages in obese individuals seem to play a 
central role. ${ }^{13}$ Like lymphocytes, adipose tissue can produce pro-inflammatory cytokines like TNF- $\alpha$ and IL- 6 and these can modulate lipid metabolism whereas TNF- $\alpha$ alters the gene expression profile of the adipocytes and the liver, resulting in increased production and release of free fatty acids, cholesterol and VLDL; elevated levels of IL-6 are associated with decreased concentration of HDL. The dysregulation of lipid metabolism by these factors may influence the development of psoriasis through regulatory effects of immune cells and keratinocytes. ${ }^{14}$ Low levels of HDL may contribute to a state of chronic inflammation. ${ }^{15}$ VLDL and LDL can increase the proliferation and expression of the LDL receptors on human keratinocytes. Both these could influence the development of psoriasis through modulation of proinflammatory cytokines in immune cells. 16

Systemic inflammation in psoriasis and an increased prevalence of unhealthy lifestyle factors have been independently associated with obesity, insulin resistance and an unfavourable cardiovascular risk profile. ${ }^{17}$ Individuals with psoriasis were more likely to have cardiovascular risk factors, including hypertension and myocardial infarction at a young age. ${ }^{18}$ Psoriasis appears to be associated with traditional risk factors for CVD, including increased Body Mass Index, hypertension, hyperlipidaemia, type 2 diabetes. ${ }^{19,20}$ Secondly, recent evidence strongly suggests that chronic inflammation, a characteristic feature of psoriasis, per se may play a role in the initiation and progression of dyslipidaemia and atherosclerosis. ${ }^{21,22}$ Elevated levels of high sensitive C-reactive protein, a nonspecific marker of inflammation is one of the emerging risk factors for cardiovascular disease. ${ }^{23,24}$

There is evidence that established treatments for psoriasis such as retinoids and cyclosporine may induce hyperlipidaemia which can promote future cardiovascular disease.25-27 Hypertriglyceridaemia secondary to VLDL elevation is associated with both procoagulant and prothrombotic factor in the blood 28 and affects the adhesiveness of platelets. ${ }^{29}$ An elevated plasma concentration of LDL cholesterol has been recognised as an important risk factor for CVD (cardiovascular disease). ${ }^{30}$ Lipoprotein (a) has similar structure to LDL, it contains an additional protein, apo (a). Lipoprotein (a) has a thrombotic effect and high levels have been linked to CVD. 31 Psoriatic arthritis may be associated with obesity, hypertension, hyperlipidaemia and insulin resistance because of the shared inflammatory pathway. ${ }^{32}$ Several cytokines that have been identified as important mediators of psoriasis such as IL-1, 4, 6, 8, 12 and TNF- $\alpha$ have also been identified in metabolic syndrome, a chronic inflammatory state associated with obesity. ${ }^{33}$ Supporting this concept is the work of Prodonowich et al, ${ }^{34}$ demonstrating that treatment of psoriasis patients with methotrexate substantially decrease the risk of cardiovascular events, especially when supplemented with folate. Higher disease severity is associated with higher cardiovascular risk as has been demonstrated in the arthritis patients. Risk was more in those who were taking systemic therapy for psoriasis. ${ }^{35}$

Severe psoriasis, diabetes mellitus, acute phase response to illness, drugs like folate antagonists - (Methotrexate), cholesterol lowering agents, thiazide, diuretics, cyclosporine can cause hyperhomocysteinaemia. ${ }^{36}$
Increased homocysteine causes vascular injury, by causing endothelial dysfunction, which is followed by platelet activation and thrombus formation. ${ }^{37}$ Genetic studies demonstrate that psoriasis and cardiovascular disease share common pathogenic features in which inflammatory cytokines - like TNF-alpha and IL-1 play an important role. Patients with psoriasis have an increased morbidity and mortality from cardiovascular events, particularly in those with severe and long duration of psoriasis disease. 38

This study was planned to screen the psoriatic patients for comorbidities (obesity, diabetes, hypertension, hyperlipidaemia), cardiovascular risk and initiate early treatment for comorbidities and to avoid cardiovascular risk.

\section{MATERIALS AND METHODS}

The prospective clinical case-control study was performed at dermatology outpatient department of Vinayaka Missions Medical College and Hospital, Karaikal. 50 clinically conformed cases of different types of psoriasis of all age groups (above 15 years) who attended the OPD between 15.10.2013 to 15.06.2014 were studied. At the same time, 20 healthy individuals without psoriasis (control) were also studied. In our study, a case of psoriasis was defined and diagnosed clinically as erythematous plaque lesions involving symmetrically the extensor aspects with positive Grattage test and Auspitz's sign.

\section{Inclusion Criteria}

1. All patients above 15 years of age who have diagnosed clinically as having psoriasis.

2. Twenty healthy individuals without psoriasis.

\section{Exclusion Criteria}

1. Non-consenting patients.

2. Patients less than 15 years of age.

3. Patients with general poor condition or infection.

A detailed history of the patients included in the study was taken, complaints, duration, evolution were noted (both skin and CVS), treatment history for the psoriasis taken. Morphology of lesion, sites of involvement noted, PASI score calculated. Family history of psoriasis, diabetic mellitus, hypertension, obesity, hyperlipidaemia taken.

\section{General and Systemic Examination Thoroughly done \\ - Body mass index calculated. \\ - Blood pressure evaluated.}

Specific Blood Investigations were done apart from the Routine Blood Investigations as follows

Random blood sugar, fasting blood sugar, postprandial blood sugar, fasting lipid profile, serum homocysteine, serum apolipoprotein, troponin, C-reactive protein, urine albumin, sugar, chest $\mathrm{x}$-ray, ECG.

\section{Reading and Interpretation of Tests}

Body mass index was calculated according to the formula weight/height ${ }^{2}$ in centimetre and obesity has interpreted if BMI is $>25$.

PASI score was calculated according to formula 0.1 $\left(E_{h}+I_{h}+D_{h}\right) A_{h}+0.2\left(E_{u}+I_{u}+D_{u}\right) A_{u}+0.3\left(E_{t}+I_{t}+D_{t}\right) A_{t}+0.4$ $\left(\mathrm{E}_{1}+\mathrm{I}_{1}+\mathrm{D}_{1}\right) \mathrm{A}_{1}$ 
PASI score $<3$ is taken as mild, moderate $-3-15$, and severe as $>15$.

Blood Pressure levels $>130 / 85$ were taken as hypertensive.

Fasting Blood sugar levels greater than $100 \mathrm{mg} / \mathrm{dL}$ were taken as abnormal.

\section{Serum Lipid Profiles were interpreted as follows}

$\begin{array}{lll} & & \text { Normal Values } \\ \text { Serum total cholesterol } & - & 150-00 \mathrm{mg} / \mathrm{dL} \\ \text { HDL } & - & 30-70 \mathrm{mg} / \mathrm{dL} \\ \text { LDL } & - & \text { up to } 120 \mathrm{mg} / \mathrm{dL} \\ \text { TGS } & - & 40-160 \mathrm{mg} / \mathrm{dL} \\ \text { VLDL } & - & 5-40 \mathrm{mg} / \mathrm{dL} \\ \text { TC/HDL Ratio } & - & 3.0 \text { low risk } \\ & & 3-5 \text { average risk } \\ & & >5 \text { high risk } \\ \text { LDL / HDL } & - & 1.5-3.5\end{array}$

\section{Criteria for Metabolic Syndrome}

\section{Obesity}

- Increased Triglycerides $\geq 150 \mathrm{mg} / \mathrm{dL}$.

- Decreased HDL - men $<40 \mathrm{mg} / \mathrm{dL}$

- Women $<50 \mathrm{mg} / \mathrm{dL}$.

- Increased Blood pressure - 130/85 mg/dL.

- Increased Fasting blood sugar - $100 \mathrm{mg} / \mathrm{dL}$.

Metabolic syndrome is identified if 3 or more of the above components are present.

Serum homocysteine levels were estimated using chemiluminescence method. The reference range was taken as $3.7-13.9 \mu \mathrm{mol} / \mathrm{L}$.

Serum apolipoprotein A1 (APO- $\mathrm{A}_{1}$ ) was calculated using Nephelometry method and the reference range was taken as $1.08-2.25 \mathrm{~g} / \mathrm{L}$.

\section{RESULTS}

Among the 50 psoriatic patients, we have encountered different varieties of psoriasis like chronic plaque psoriasis in 34, chronic plaque psoriasis with arthritis in 6, pustular psoriasis in 5 , Guttate psoriasis in 4 , erythrodermic in 1 . In our study out of 50 patients, 32 (64\%) were males, $18(36 \%)$ were females. More number of psoriatic patients (15) were between 41-50 years of age group. The positive cases (with HTN, DM, obesity and hyperlipidaemia) are more in the age group of $51-60(38.88 \%)$. Out of HTN, DM, obesity and hyperlipidaemia, any 2 were positive in one case. $(2.79 \%)$, any 3 were positive in 14 cases (38.88\%), and all 4 were positive in 21 cases (58.33\%). According to the metabolic syndrome criteria, we can analyse from the above that almost 35 patients out of 50 psoriatic patients are suffering from metabolic syndrome and at high cardiovascular risk. Among controls, one was suffering from metabolic syndrome. When compared to control, the p-value was $<0.05$ and was significant for BMI (body mass index), blood sugars, hypertension.

\begin{tabular}{|c|c|c|c|}
\hline Psoriasis Cases & Total & $\begin{array}{c}\text { Positive } \\
\text { Results }\end{array}$ & $\begin{array}{c}\text { Negative } \\
\text { Results }\end{array}$ \\
\hline $\begin{array}{c}\text { Number of } \\
\text { Patients (N) }\end{array}$ & 50 & 36 & 14 \\
\hline Percentage $\%$ & $100 \%$ & $72 \%$ & $28 \%$ \\
\hline \multicolumn{3}{|c|}{ Table 1. Tested Patients } \\
\hline
\end{tabular}

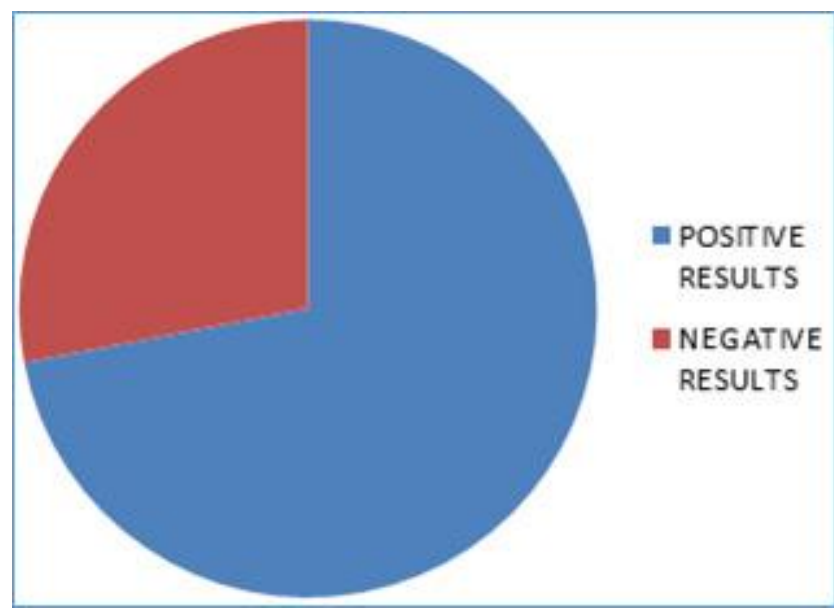

\begin{tabular}{|c|c|c|}
\hline Sex & Frequency & Percentage \\
\hline Female & 18 & $36.00 \%$ \\
\hline Male & 32 & $64 \%$ \\
\hline Total & $\mathbf{5 0}$ & $\mathbf{1 0 0} \%$ \\
\hline \multicolumn{2}{|c|}{ Table 2. Sex Distribution of the Patients } \\
\hline
\end{tabular}

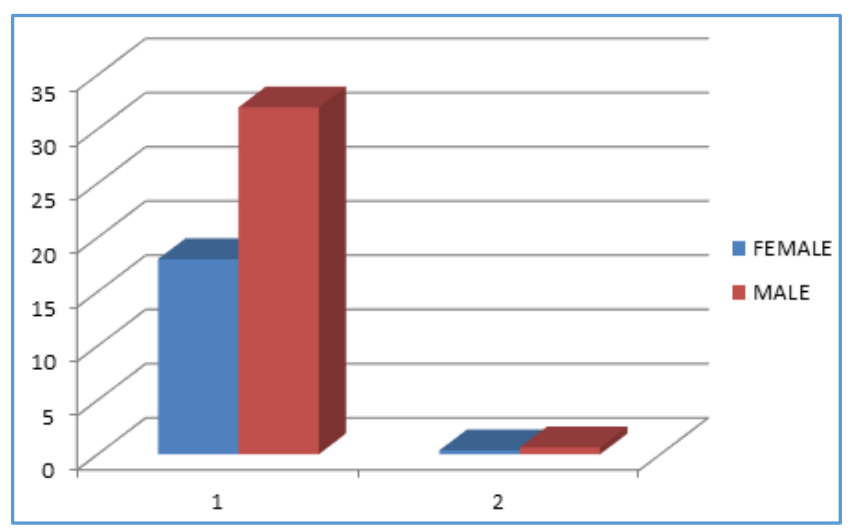

\begin{tabular}{|c|c|c|}
\hline Age & Frequency & Percentage \\
\hline 21- 30 Years & 2 & $4.0 \%$ \\
\hline 31-40 Years & 11 & $22.0 \%$ \\
\hline 41-50 Years & 15 & $30.0 \%$ \\
\hline 51- 60 Years & 14 & $28.0 \%$ \\
\hline 61-70 Years & 7 & $14.0 \%$ \\
\hline 71-80 Years & 1 & $2.0 \%$ \\
\hline Total & 50 & $100.00 \%$ \\
\hline \multicolumn{3}{|c|}{ Table 3. Age Distribution of the Patients } \\
\hline
\end{tabular}



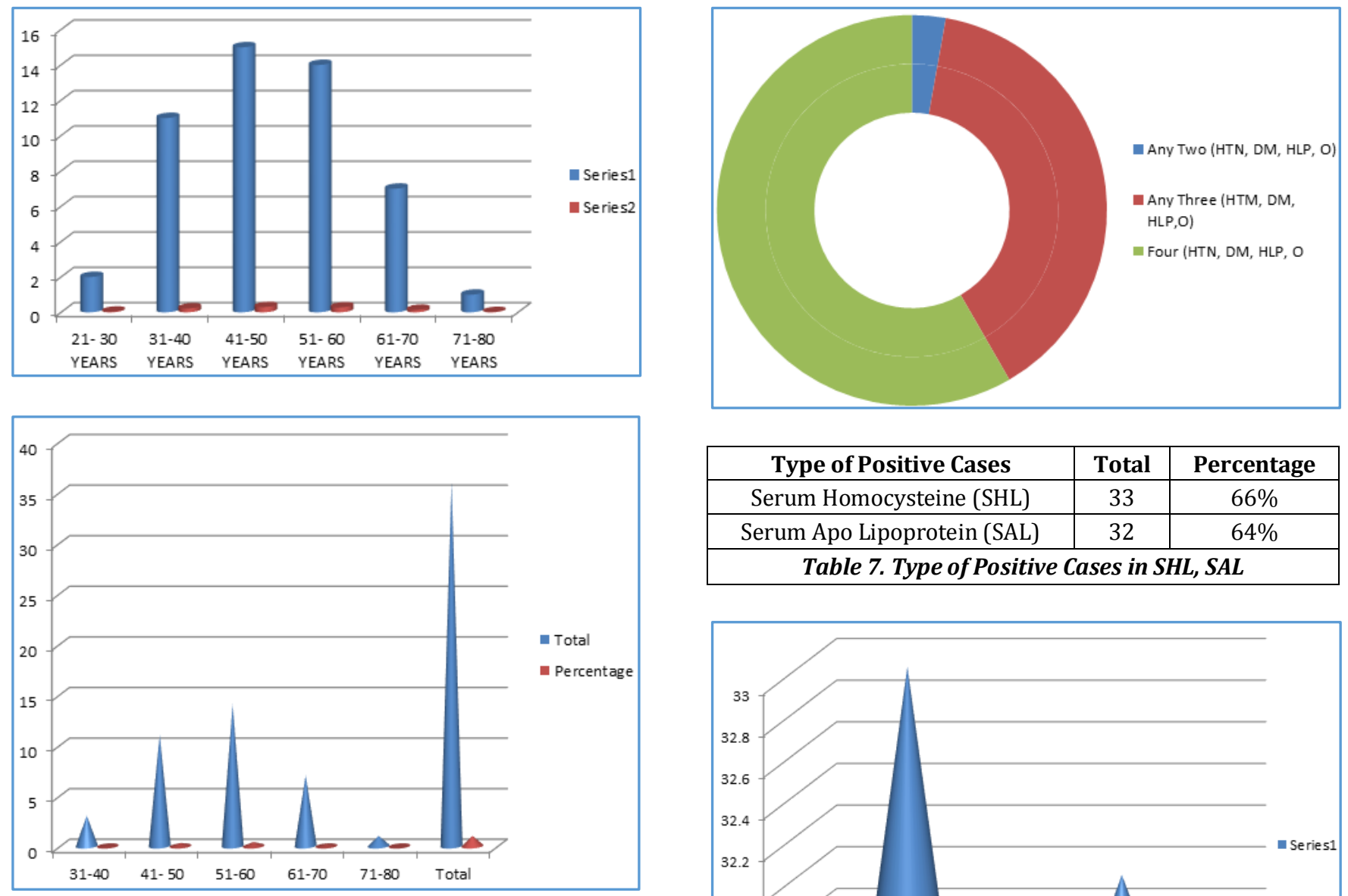

\begin{tabular}{|c|c|c|}
\hline Type of Positive Cases & Total & Percentage \\
\hline Serum Homocysteine (SHL) & 33 & $66 \%$ \\
\hline Serum Apo Lipoprotein (SAL) & 32 & $64 \%$ \\
\hline \multicolumn{2}{|c|}{ Table 7. Type of Positive Cases in SHL, SAL } \\
\hline
\end{tabular}

\begin{tabular}{|c|c|c|}
\hline Individual Cases & Total & Percentage \\
\hline Hypertension (HTN) & 31 & $62 \%$ \\
\hline Diabetes Mellitus (DM) & 31 & $62 \%$ \\
\hline Hyperlipidaemia (HLP) & 35 & $70 \%$ \\
\hline Obesity (0) & 33 & $66 \%$ \\
\hline \multicolumn{3}{|c|}{$\begin{array}{c}\text { Table 5. Type of Individual Positive Cases } \\
(H T N, D M, H L P, O)\end{array}$} \\
\hline
\end{tabular}
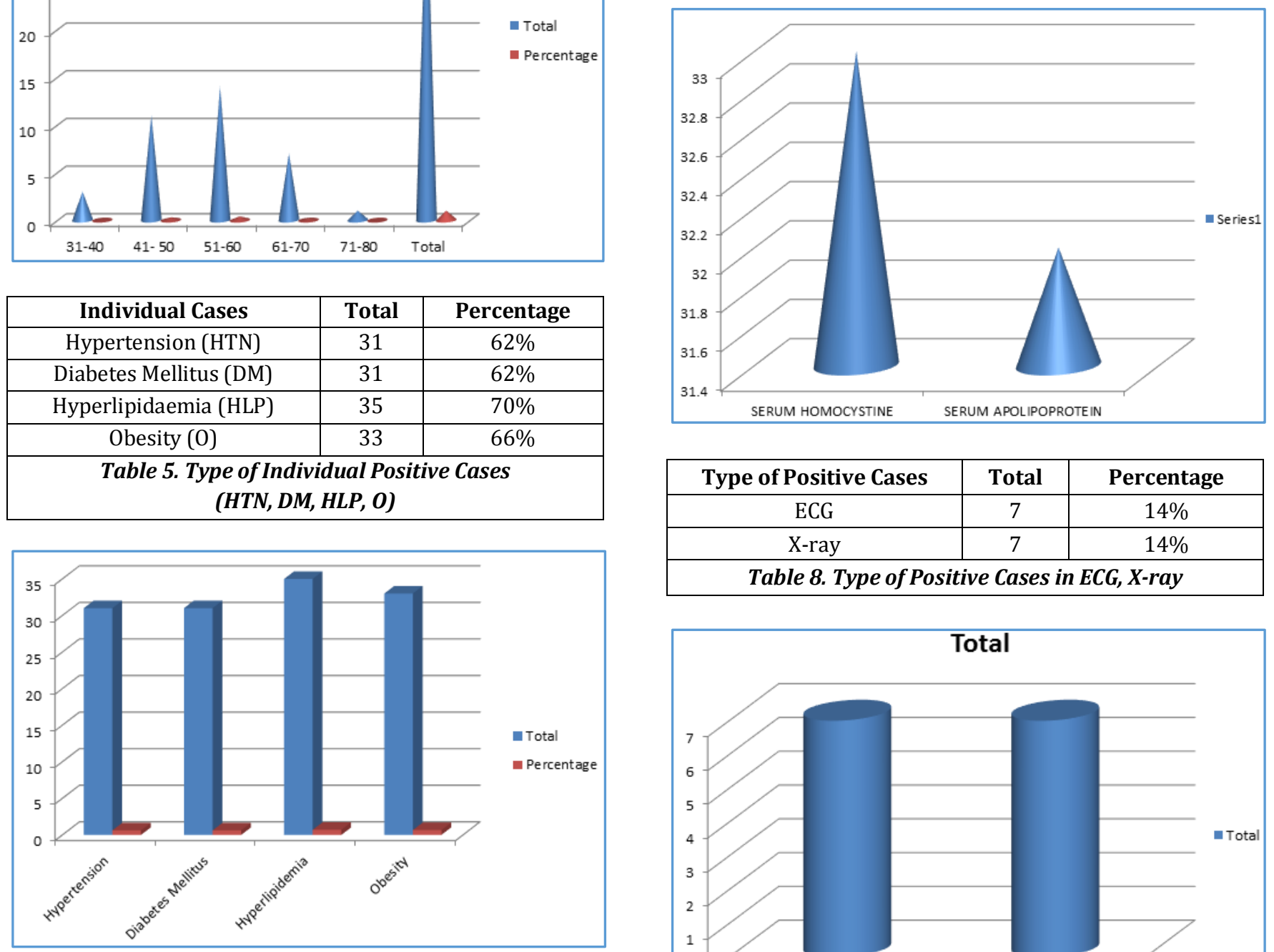

\begin{tabular}{|c|c|c|}
\hline Type of Positive Cases & Total & Percentage \\
\hline ECG & 7 & $14 \%$ \\
\hline X-ray & 7 & $14 \%$ \\
\hline \multicolumn{2}{|c|}{ Table 8. Type of Positive Cases in ECG, X-ray } \\
\hline
\end{tabular}

\begin{tabular}{|c|c|c|}
\hline Individual Cases & Total & Percentage \\
\hline Any Two (HTN, DM, HLP, O) & 1 & $2.79 \%$ \\
\hline Any Three (HTM, DM, HLP, O) & 14 & $38.88 \%$ \\
\hline Four (HTN, DM, HLP, O) & 21 & $58.33 \%$ \\
\hline \multicolumn{2}{|c|}{ Table 6. Single/Multiple Positive Cases } \\
(HTN, DM, HLP, o)
\end{tabular}

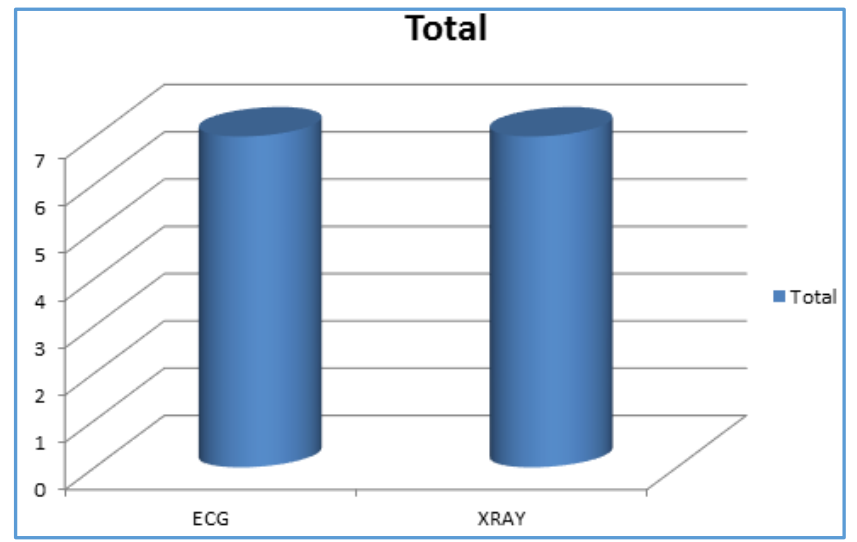

\begin{tabular}{|c|c|c|}
\hline Type of Positive Cases & Total & Percentage \\
\hline Urine sugar & 28 & $56 \%$ \\
\hline Urine albumin & 16 & $32 \%$ \\
\hline \multicolumn{2}{|c|}{ Table 9. Type of Positive Cases in US, UA } \\
\hline
\end{tabular}



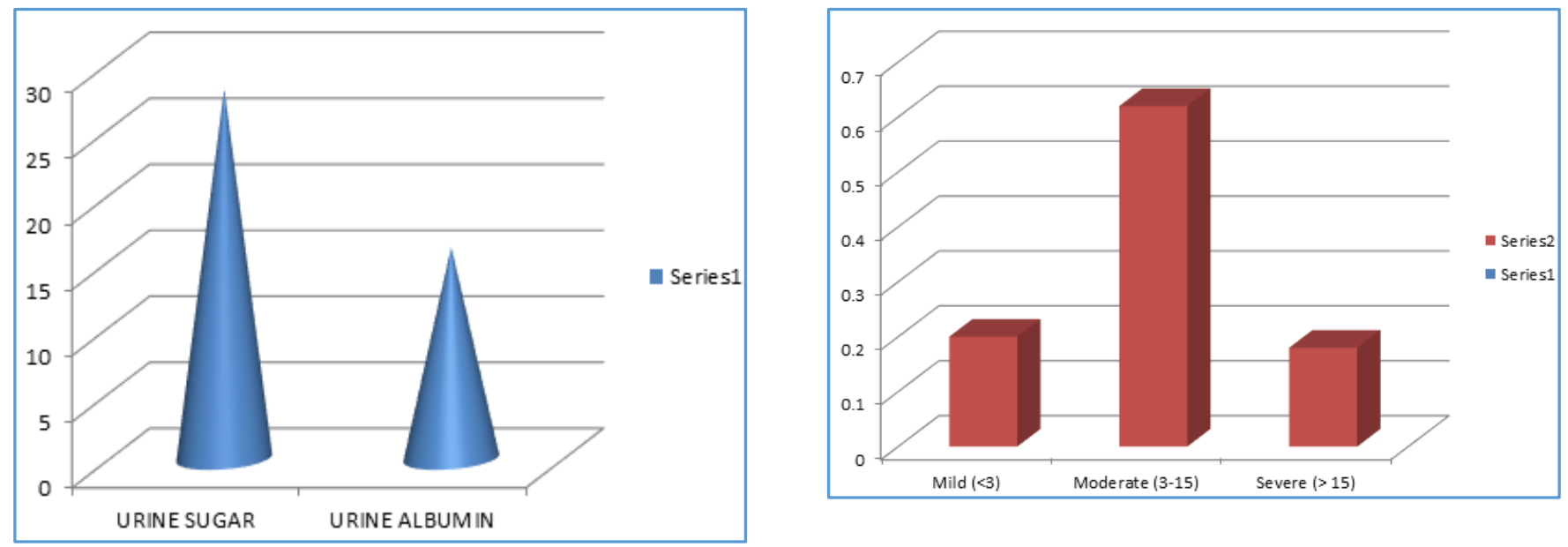

\begin{tabular}{|c|c|c|c|c|}
\hline & 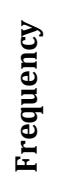 & de & ত & $\underbrace{\stackrel{\Xi}{\Xi}}$ \\
\hline Valid 0-5 Years & 15 & $30 \%$ & 30.0 & 30.0 \\
\hline 6-10 Years & 15 & $30 \%$ & 30.0 & 60.0 \\
\hline 11-15 Years & 14 & $28 \%$ & 28.0 & 88.0 \\
\hline 16-20 Years & 4 & $8 \%$ & 8.0 & 96.0 \\
\hline 21-25 Years & 2 & $4 \%$ & 4.0 & 100.0 \\
\hline Total & 5 & $100 \%$ & 100.0 & \\
\hline \multicolumn{5}{|c|}{ Table 10. Duration of Psoriasis of the Patients } \\
\hline
\end{tabular}

\begin{tabular}{|c|c|c|}
\hline $\begin{array}{c}\text { Duration of } \\
\text { Psoriasis } \\
\text { (Years) }\end{array}$ & $\begin{array}{c}\text { Duration of } \\
\text { Comorbidities } \\
\text { (Years) }\end{array}$ & $\begin{array}{c}\text { Duration of CVS } \\
\text { Complaints (MI, } \\
\text { Angina, } \\
\text { Arrhythmias) } \\
\end{array}$ \\
\hline 10 & 4 & $\begin{array}{l}\text { L.A.E } 2 \text { YEAR (Left } \\
\text { atrial enlargement) }\end{array}$ \\
\hline 15 & 8 & \\
\hline 10 & 3 & $\begin{array}{c}\text { L.V.H. } 1 \text { YEAR (left } \\
\text { ventricular } \\
\text { hypertrophy) } \\
\end{array}$ \\
\hline 15 & 8 & \\
\hline 15 & 6 & \\
\hline 14 & 5 & \\
\hline 15 & 8 & L.V.H 5 YEARS \\
\hline 22 & 6 & \\
\hline 6 & 1 & \\
\hline 12 & 7 & \\
\hline 7 & 2 & \\
\hline 7 & 3 & \\
\hline 5 & 1 & \\
\hline 14 & 6 & \\
\hline 11 & 4 & \\
\hline 18 & 10 & \\
\hline 9 & 3 & \\
\hline 13 & 6 & $\begin{array}{c}\text { L.V.SOL } 4 \text { YEARS (left } \\
\text { ventricular systolic } \\
\text { overload) }\end{array}$ \\
\hline 16 & 7 & \\
\hline 20 & 8 & L.V.H 6 YEARS \\
\hline 22 & 10 & \\
\hline 15 & 6 & \\
\hline 8 & 2 & \\
\hline 10 & 3 & \\
\hline 11 & 4 & L.A.E 3 YEARS \\
\hline 6 & 1 & \\
\hline 9 & 2 & \\
\hline 13 & 5 & \\
\hline 15 & 6 & \\
\hline 9 & 2 & \\
\hline 6 & 1 & \\
\hline 7 & 1 & L.V.SOL \\
\hline 16 & 4 & \\
\hline 15 & 5 & \\
\hline 7 & 2 & \\
\hline 8 & 1 & \\
\hline \multicolumn{3}{|c|}{ Table 12. Duration of Concerned Illnesses } \\
\hline
\end{tabular}




\begin{tabular}{|c|c|}
\hline Type of Psoriasis & Total \\
\hline Chronic Plaque Psoriasis & 34 \\
\hline Pustular Psoriasis & 5 \\
\hline Chronic Plaque Psoriasis with arthritis & 6 \\
\hline Guttate type of Psoriasis & 6 \\
\hline Psoriatic Erythroderma & 1 \\
\hline Table 13. Types of Psoriasis Encountered \\
\hline
\end{tabular}
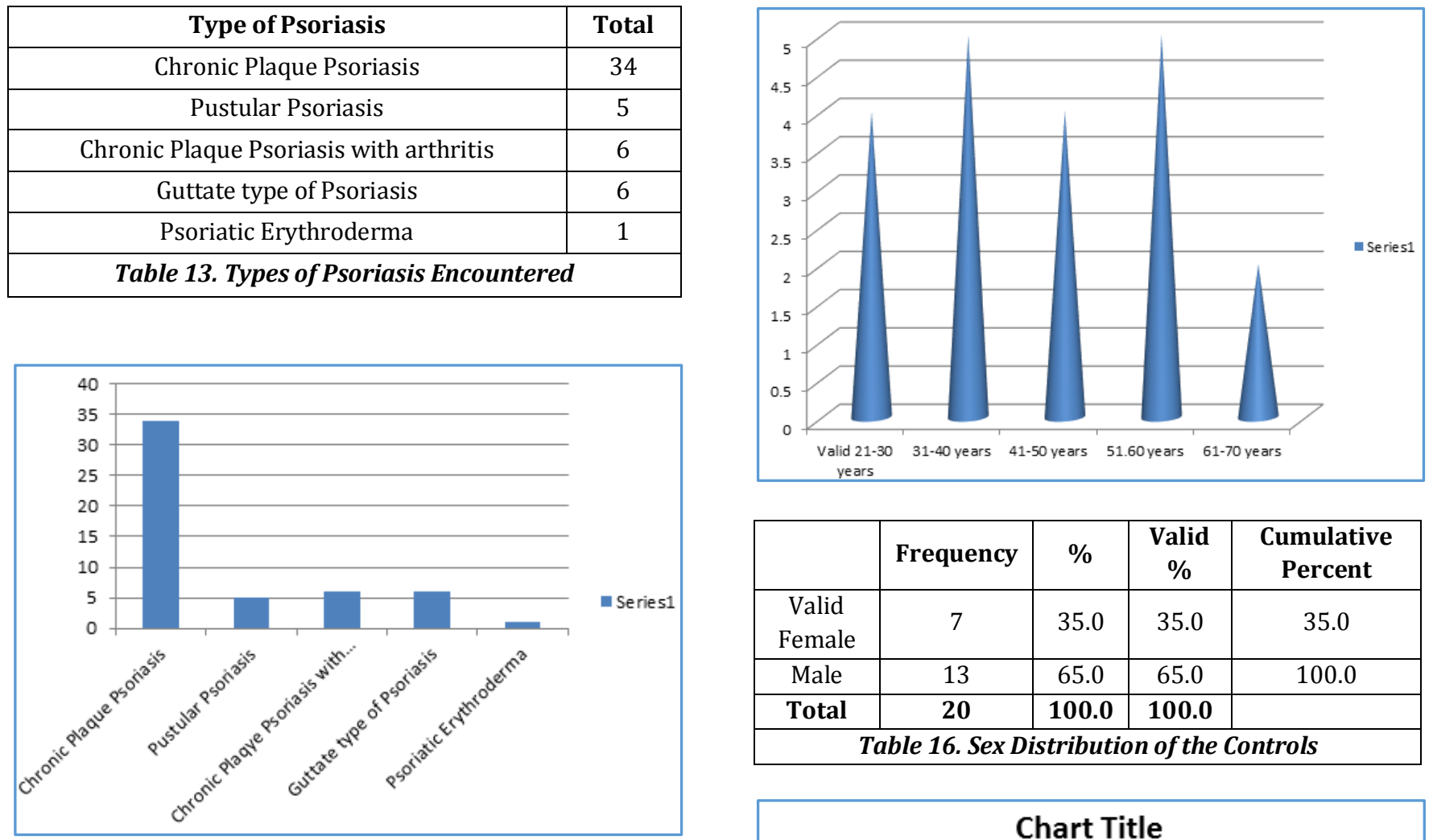

\begin{tabular}{|c|c|c|c|c|}
\hline & Frequency & $\mathbf{\%}$ & $\begin{array}{c}\text { Valid } \\
\%\end{array}$ & $\begin{array}{c}\text { Cumulative } \\
\text { Percent }\end{array}$ \\
\hline $\begin{array}{c}\text { Valid } \\
\text { Female }\end{array}$ & 7 & 35.0 & 35.0 & 35.0 \\
\hline Male & 13 & 65.0 & 65.0 & 100.0 \\
\hline Total & 20 & $\mathbf{1 0 0 . 0}$ & $\mathbf{1 0 0 . 0}$ & \\
\hline \multicolumn{4}{|c|}{ Table 16. Sex Distribution of the Controls } \\
\hline
\end{tabular}

\begin{tabular}{|c|c|c|c|}
\hline Controls & Total & +ve Results & -ve Results \\
\hline $\begin{array}{c}\text { Number of } \\
\text { Patients (N) }\end{array}$ & 20 & 6 & 14 \\
\hline Percentage $\%$ & $\begin{array}{c}100 \\
\%\end{array}$ & $30 \%$ & $70 \%$ \\
\hline \multicolumn{4}{|c|}{ Table 14. Controls } \\
\hline
\end{tabular}
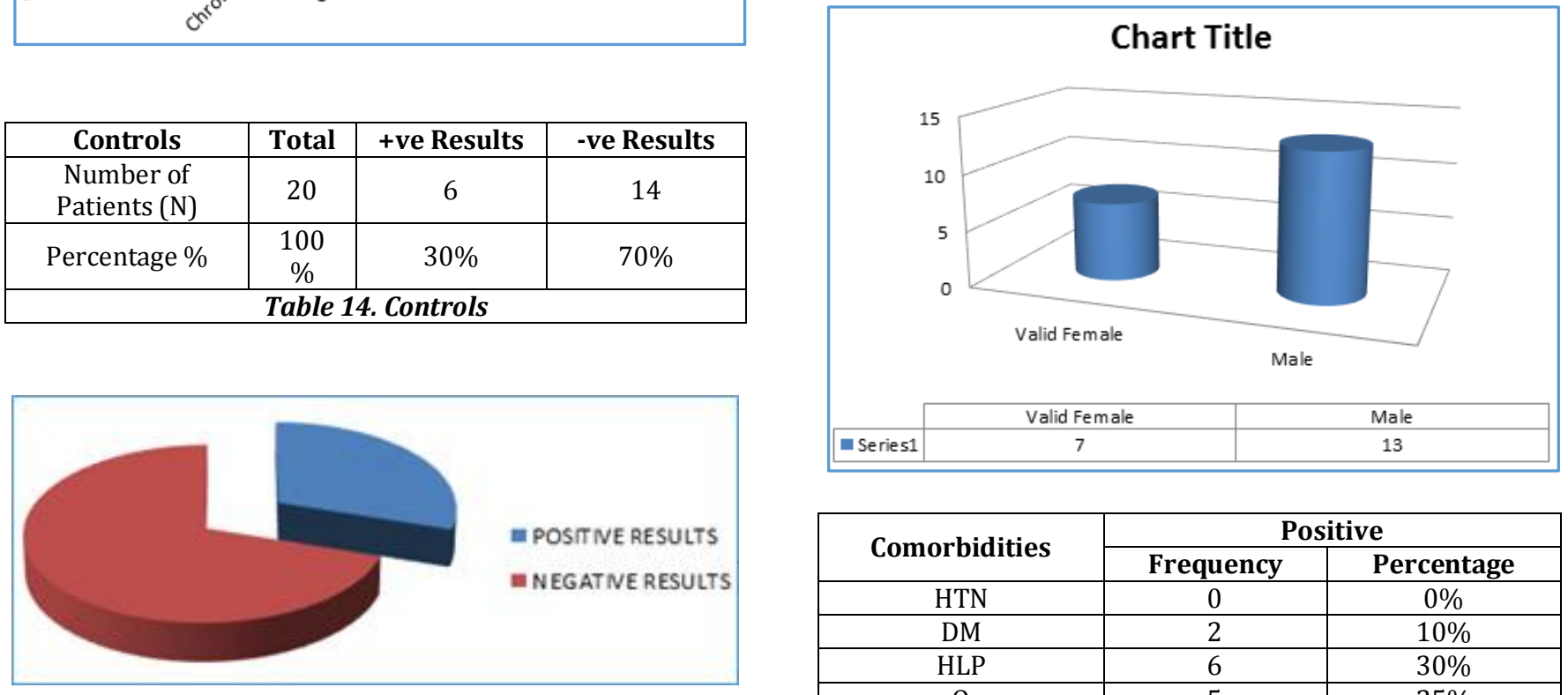

\begin{tabular}{|c|c|c|}
\hline \multirow{2}{*}{ Comorbidities } & \multicolumn{2}{|c|}{ Positive } \\
\cline { 2 - 3 } & Frequency & Percentage \\
\hline HTN & 0 & $0 \%$ \\
\hline DM & 2 & $10 \%$ \\
\hline HLP & 6 & $30 \%$ \\
\hline O & 5 & $25 \%$ \\
\hline Table 17. Individual Cases of the Positive Cases (Controls) \\
\hline
\end{tabular}

\begin{tabular}{|c|c|c|c|c|}
\hline & 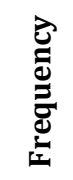 & 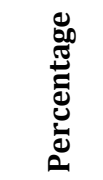 & $\frac{\overbrace{}^{0}}{\frac{\pi}{7}}$ & 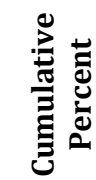 \\
\hline Valid $21-30$ years & 4 & 20.0 & 20.0 & 20.0 \\
\hline $31-40$ years & 5 & 25.0 & 25.0 & 45.0 \\
\hline 41-50 years & 4 & 20.0 & 20.0 & 65.0 \\
\hline 51.60 years & 5 & 25.0 & 25.0 & 90.0 \\
\hline 61-70 years & 2 & 10.0 & 10.0 & 100.0 \\
\hline Total & 20 & 100.0 & 100.0 & \\
\hline \multicolumn{5}{|c|}{ Table 15. Age Distribution of the Controls } \\
\hline
\end{tabular}

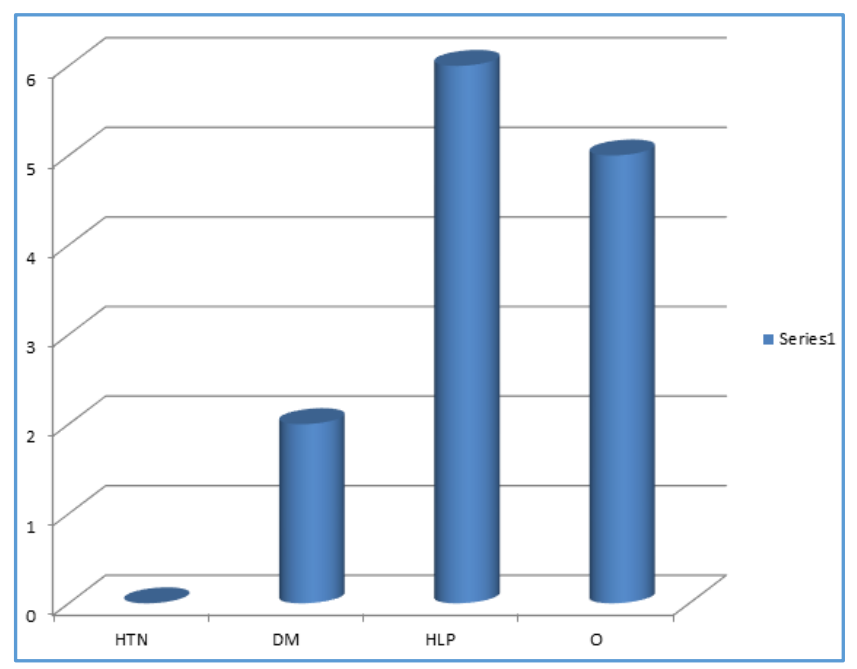




\begin{tabular}{|c|c|c|c|c|}
\hline VAR00001 & N & Mean & Std. Deviation & Std. Error Mean \\
\hline BMI 1 & 50 & 25.6992 & 1.64813 & .23308 \\
2 & 20 & 23.5635 & 1.62260 & .36282 \\
\hline \multicolumn{4}{|c|}{ Table 18. Comparing Two Independent means - Psoriasis Patients and Normal Patients for BMI } \\
Group Statistics
\end{tabular}

Independent Sample Test

\begin{tabular}{|c|c|c|c|c|c|c|c|c|c|}
\hline & \multicolumn{2}{|c|}{$\begin{array}{l}\text { Levene's Test for } \\
\text { Equality of } \\
\text { Variances }\end{array}$} & \multicolumn{7}{|c|}{ t- Test for Equality of Means } \\
\hline & \multirow[t]{2}{*}{$\mathbf{F}$} & \multirow[t]{2}{*}{ Sig } & \multirow[t]{2}{*}{$\mathbf{T}$} & \multirow[t]{2}{*}{ Df } & \multirow[t]{2}{*}{$\begin{array}{l}\text { Sig.(2- } \\
\text { tailed) }\end{array}$} & \multirow[t]{2}{*}{$\begin{array}{c}\text { Mean } \\
\text { Difference }\end{array}$} & \multirow[t]{2}{*}{$\begin{array}{l}\text { Std. Error } \\
\text { Difference }\end{array}$} & \multicolumn{2}{|c|}{$\begin{array}{c}95 \% \\
\text { Confidence Interval of } \\
\text { the Difference }\end{array}$} \\
\hline & & & & & & & & Lower & Upper \\
\hline $\begin{array}{c}\text { BMI Equal } \\
\text { Variances } \\
\text { Assumed } \\
\text { Equal } \\
\text { variances not } \\
\text { assumed }\end{array}$ & .010 & .922 & $\begin{array}{l}4.919 \\
4.952\end{array}$ & $\begin{array}{c}68 \\
35.569\end{array}$ & $\begin{array}{l}0.00 \\
0.00\end{array}$ & $\begin{array}{l}2.13570 \\
2.13570\end{array}$ & $\begin{array}{l}.43418 \\
.43124\end{array}$ & $\begin{array}{l}1.26931 \\
1.26074\end{array}$ & $\begin{array}{l}3.00209 \\
3.01066\end{array}$ \\
\hline
\end{tabular}

$\mathrm{P}$ Value $<0.05$, Tested value 0.000 , Tested Value $<0.05$, so $\mathrm{P}$ value is significant

\begin{tabular}{|c|c|c|c|c|}
\hline VAR00001 & N & Mean & Std. Deviation & Std. Error Mean \\
\hline Blood sugar 1 & 50 & 159.66 & 34.281 & 4.848 \\
2 & 20 & 125.65 & 20.841 & 4.660 \\
\hline
\end{tabular}

Independent Sample Test

\begin{tabular}{|c|c|c|c|c|c|c|c|c|c|}
\hline & \multicolumn{2}{|c|}{$\begin{array}{c}\text { Levene's Test for } \\
\text { Equality of Variances }\end{array}$} & \multicolumn{7}{|c|}{ t- test for Equality of Means } \\
\hline & \multirow[t]{2}{*}{$\mathbf{F}$} & \multirow[t]{2}{*}{ Sig } & \multirow[t]{2}{*}{$\mathbf{T}$} & \multirow[t]{2}{*}{ Df } & \multirow[t]{2}{*}{$\begin{array}{l}\text { Sig.(2- } \\
\text { Tailed) }\end{array}$} & \multirow[t]{2}{*}{$\begin{array}{c}\text { Mean } \\
\text { Difference }\end{array}$} & \multirow[t]{2}{*}{$\begin{array}{l}\text { Std. Error } \\
\text { Difference }\end{array}$} & \multicolumn{2}{|c|}{$\begin{array}{c}95 \% \\
\text { Confidence Interval of } \\
\text { the Difference }\end{array}$} \\
\hline & & & & & & & & Lower & Upper \\
\hline $\begin{array}{c}\text { Blood } \\
\text { sugar } \\
\text { Equal } \\
\text { Variances } \\
\text { Assumed } \\
\text { Equal } \\
\text { Variances } \\
\text { not } \\
\text { assumed }\end{array}$ & 3.263 & 0.075 & $\begin{array}{l}4.131 \\
5.057\end{array}$ & $\begin{array}{c}68 \\
56.651\end{array}$ & $\begin{array}{l}0.00 \\
0.00\end{array}$ & $\begin{array}{l}34.010 \\
34.010\end{array}$ & $\begin{array}{l}8.233 \\
6.725\end{array}$ & $\begin{array}{l}17.582 \\
20.542\end{array}$ & $\begin{array}{l}50.438 \\
47.478\end{array}$ \\
\hline
\end{tabular}

P Value $<0.05$, Tested value 0.000 , Tested Value $<0.05$, so $\mathrm{P}$ value is significant.

\begin{tabular}{|c|c|c|c|c|}
\hline VAR00001 & N & Mean & Std. Deviation & Std. Error Mean \\
\hline BP Says 1 & 50 & 142.00 & 20.102 & 2.843 \\
2 & 20 & 121.50 & 14.244 & 3.185 \\
\hline BP Dia 1 & 50 & 88.80 & 11.891 & 2.682 \\
2 & 20 & 82.00 & 8.944 & 000 \\
\hline \multicolumn{2}{|r}{ Table 20. Comparing Two Independent means Psoriasis Patients and Normal Patients for Hypertension } \\
\end{tabular}


Independent Sample Test

\begin{tabular}{|c|c|c|c|c|c|c|c|c|c|}
\hline & \multicolumn{2}{|c|}{$\begin{array}{c}\text { Levene's Test for } \\
\text { Equality of } \\
\text { Variances } \\
\end{array}$} & \multicolumn{7}{|c|}{ t- test for Equality of Means } \\
\hline & \multirow[t]{2}{*}{$\mathbf{F}$} & \multirow[t]{2}{*}{ Sig } & \multirow[t]{2}{*}{$\mathbf{T}$} & \multirow[t]{2}{*}{ Df } & \multirow[t]{2}{*}{$\begin{array}{l}\text { Sig.(2- } \\
\text { tailed) }\end{array}$} & \multirow[t]{2}{*}{$\begin{array}{c}\text { Mean } \\
\text { Difference }\end{array}$} & \multirow[t]{2}{*}{$\begin{array}{l}\text { Std. Error } \\
\text { Difference }\end{array}$} & \multicolumn{2}{|c|}{$\begin{array}{c}95 \% \\
\text { Confidence Interval } \\
\text { of the Difference }\end{array}$} \\
\hline & & & & & & & & Lower & Upper \\
\hline $\begin{array}{c}\text { Equal } \\
\text { Variances } \\
\text { Assumed } \\
\text { Equal } \\
\text { variances not } \\
\text { assumed }\end{array}$ & 4.828 & 0.031 & $\begin{array}{l}4.154 \\
4.802\end{array}$ & $\begin{array}{c}68 \\
49.218\end{array}$ & $\begin{array}{l}000 \\
000\end{array}$ & $\begin{array}{l}20.500 \\
20.500\end{array}$ & $\begin{array}{l}4.935 \\
4.269\end{array}$ & $\begin{array}{l}10.653 \\
11.922\end{array}$ & $\begin{array}{l}30.347 \\
29.078\end{array}$ \\
\hline $\begin{array}{c}\text { Equal } \\
\text { Variances } \\
\text { Assumed }\end{array}$ & .180 & .672 & 2.306 & 68 & .024 & 6.800 & 2.949 & .915 & 12.685 \\
\hline
\end{tabular}

\section{Independent Sample Test}

\begin{tabular}{|c|c|c|c|c|c|c|c|c|c|}
\hline & \multicolumn{2}{|c|}{$\begin{array}{c}\text { Levene's Test for } \\
\text { Equality of } \\
\text { Variances } \\
\end{array}$} & \multicolumn{7}{|c|}{ t- Test for Equality of Means } \\
\hline & \multirow[t]{2}{*}{$\mathbf{F}$} & \multirow[t]{2}{*}{ Sig } & \multirow[t]{2}{*}{$\mathbf{T}$} & \multirow[t]{2}{*}{ Df } & \multirow[t]{2}{*}{$\begin{array}{l}\text { Sig.(2- } \\
\text { tailed) }\end{array}$} & \multirow[t]{2}{*}{$\begin{array}{c}\text { Mean } \\
\text { Difference }\end{array}$} & \multirow[t]{2}{*}{$\begin{array}{l}\text { Std. Error } \\
\text { Difference }\end{array}$} & \multicolumn{2}{|c|}{$\begin{array}{c}95 \% \\
\text { Confidence Interval } \\
\text { of the Difference }\end{array}$} \\
\hline & & & & & & & & Lower & Upper \\
\hline $\begin{array}{c}\text { Equal } \\
\text { Variances } \\
\text { Assumed } \\
\text { Equal } \\
\text { variances not } \\
\text { assumed }\end{array}$ & 4.828 & 0.031 & $\begin{array}{l}4.154 \\
4.802\end{array}$ & $\begin{array}{c}68 \\
49.218\end{array}$ & $\begin{array}{l}000 \\
000\end{array}$ & $\begin{array}{l}20.500 \\
20.500\end{array}$ & $\begin{array}{l}4.935 \\
4.269\end{array}$ & $\begin{array}{l}10.653 \\
11.922\end{array}$ & $\begin{array}{l}30.347 \\
29.078\end{array}$ \\
\hline $\begin{array}{c}\text { Equal } \\
\text { Variances } \\
\text { Assumed }\end{array}$ & .180 & .672 & 2.306 & 68 & .024 & 6.800 & 2.949 & .915 & 12.685 \\
\hline $\begin{array}{c}\text { Equal } \\
\text { Variances not } \\
\text { Assumed }\end{array}$ & & & 2.602 & 46.373 & 0.012 & 6.800 & 2.613 & 1.541 & 12.059 \\
\hline
\end{tabular}

B.P.Sys, P Value $<0.05$; Tested Value 0.000 , Tested value $<0.05$, so P value is significant, B.P.Dia, P Value $<0.05 ;$ Tested Value 0.024 Tested value $<0.05$, so $P$ value is significant.

\section{DISCUSSION}

Psoriasis is a chronic inflammatory autoimmune disease. The pathophysiology of psoriasis is characterised by an increase in antigen presentation, $\mathrm{T}$ cell activation and $\mathrm{T}$-helper cell type $1\left(\mathrm{~T}_{\mathrm{H} 1}\right)$ cytokines, resulting in thick scaly red plaques and in some patients' arthritis.

The immunological abnormalities that lead to the development of psoriasis suggests that these patients may be at increased risk for other diseases associated with an inflammatory state.

A $\mathrm{T}_{\mathrm{H} 1}$ immune response including activated $\mathrm{T}$ cells, antigen presenting cells, cytokines are important to the development of atherosclerosis and ultimately myocardial infraction(MI).
In our study, there was a more male dominance correlating with the previous studies. ${ }^{2}$

All the varieties of psoriasis are associated with comorbidities irrespective of the severity of the disease correlating with the previous studies.

In our study, metabolic syndrome is seen in patients who are older and having a longer duration of the disease when compared to psoriasis patients without metabolic syndrome. Our study demonstrated increased risk of hypertension, diabetes mellitus, altered lipid profiles and obesity in psoriasis, consistent with the previous studies. 4,5,6,7

Risk of diabetes mellitus among individuals with psoriasis has been shown in previous studies consistent with our prospective study. 
The psoriatic patients with diabetes in our study were using longterm topical steroids on large body surface areas which could explain the risk for diabetes which is in accordance with the previous studies. ${ }^{7}$

An increased risk of hypertension has been reported in previous studies.7,8,9

In our study, individuals with psoriasis were also at increased risk of hypertension. Potential explanation for this association include systemic inflammation and psoriasis treatment.

In our study, a female patient with pustular psoriasis treated with methotrexate, cyclosporine and retinoids consequently developed hypertension, diabetes and hyperlipidaemia. Whether these comorbidities are due to psoriasis per se or due to the treatments given has to be determined.

Among 20 controls investigated, six were found to have metabolic disease among which 1 was having diabetes, hyperlipidaemia and obesity; 1 was having diabetes mellitus and hyperlipidaemia and obesity; 1 was having diabetes mellitus and hyperlipidaemia. These findings can determine some other ailments which can predispose to cardiovascular risk and psoriasis is not the only independent risk factor for cardiovascular morbidity.

In our study, psoriasis preceded the metabolic diseases (syndrome) and cardiovascular complaints in all the cases, which is not in accordance with the previous studies. ${ }^{4,5,6}$ Whether metabolic syndrome per se can predispose to psoriasis has to be discussed.

Prevalence of metabolic syndrome and metabolic diseases like hypertension, diabetes, obesity and hyperlipidaemia were more in psoriatics when compared to controls.

\section{In my Study out of 50 Patients:}

- $32(64 \%)$ were males.

- 18 (36\%) were females.

More number of psoriatic patients (15) were between 41 50 years of age group.

The positive cases (HTN, DM, Obesity and Hyperlipidaemia) (14 cases) are more in the age group 51-60 $(38.88 \%)$. In our study, we could not get to study more no. of patients in younger age group (Only 2 between 21-30) and these patients were not found to have hypertension, diabetes and hyperlipidaemias which is not in accordance with the previous studies. 18

Serum homocysteine levels were raised in $66 \%$ of cases which is an increased risk factor for cardiovascular disease and this finding is consistent with the previous studies. ${ }^{37}$

Out of HTN, DM, obesity and hyperlipidaemia, any two were positive in one case (2.79\%), any three were positive in 14 cases $(38.88 \%)$ and all four were positive in 21 cases(58.33\%). According to the metabolic syndrome criteria, we can analyse from the above that almost 35 patients out of 50 psoriatic patients are suffering from metabolic syndrome. Among controls, 1 was suffering from metabolic syndrome.

When compared to controls, the $p$ value was $<0.05$ and was significant for BMI, Blood sugar, Hypertension.

\section{CONCLUSION}

With this we can analyse that psoriasis is a definite risk for metabolic syndrome and cardiovascular morbidity and most of our findings were consistent with the previous studies.

\section{REFERENCES}

1. Pavithran K, Karunakaran K, Palit A, et al. Disorders of keratinisation. In: Valia RG, Valia AR. IADVL textbook of dermatology. $3^{\text {rd }}$ edn. Bhalari publications \& Co 2008:1021-22.

2. Lal S. Clinical pattern of psoriasis in Punjab. Indian J Dermatol Venereol 1966;35:5-12.

3. Pavithran K, Karunakaran K, Palit A, et al. Disorders of keratinisation. In: Valia RG, Valia AR. IADVL textbook of dermatology. $3^{\text {rd }}$ edn. Bhalari publications \& Co 2008:1021-24.

4. Mallbris L, Granath F, Hamsten A, et al. Psoriasis is associated with lipid abnormalities at the onset of skin disease. J Am Acad Dermatol 2006;54(4):614-21.

5. Rocha-Pereira P, Santos-Silva A, Rebelo I, et al. Dyslipidemia and oxidative stress in mild and in severe psoriasis as risk for cardiovascular disease. Clint chim Acta 2001;303(1-2):33-9.

6. Cimsit G, Orem A, Deger 0, et al. The variations of serum apolipoprotein-alpha with disease activity in psoriasis. British journal of dermatology 1988;138(5):917-9.

7. Lindegard B. Diseases associated with psoriasis in general population of 159,00 middle-aged, urban, native Swedes. Dermatologica 1986;172(6):298-304.

8. Das UN. Is angiotensin-II an endogenous proinflammatory molecule? Med Sci Monit 2005;11(5):RA155-62.

9. Ucak S, Ekmekci TR, Basat O, et al. Comparison of various insulin sensitivity indices in psoriatic patients and their relationship with type of psoriasis. J Eur Acad Dermatol Venereol 2006;20(5):517-22.

10. Chodorowska G, Wojnowska D, Juszkiewicz-Borowiec M. C-reactive protein and alpha2-macroglobulin plasma activity in medium-severe and severe psoriasis. J Eur Acad Dermatol Venereol 2004;18(2):180-3.

11. Rocha-Pereira P, Santo-Silva A, Rebelo I, et al. The inflammatory response in mild and severe psoriasis. Br J Dematol 2004;150(5):917-28.

12. Ridker PM, Rifai N, Cook NR, et al. Non-HDL cholesterol, apolipoprotein A-I and B-100, standard lipid measures, lipid ratios and CRP as risk factors for cardiovascular disease in women. JAMA 2005;294(3):326-33.

13. Lee YH, Prateley RE. The evolving role of inflammation in obesity and the metabolic syndrome. Curr Diab Rep 2005;5(1):70-5.

14. Ruan H, Miles PD, Ladd CM, et al. Profiling gene transcription in vivo reveals adipose tissue as intermediate target of tumour necrosis factor alpha: implication for insulin resistance. Diabetes 2002;51:317688.

15. Borger D, Dayer JM. High-density lipoprotein-associated apolipoprotein A-I: the missing link between infection and chronic inflammation? Autoimmune Rev 2002;1(12):111-7.

16. Furomoto H, Arai H, Kuramitsu Y, et al. Lipoproteins modulate growth and differentiation of cultured human epidermal keratinocytes. Electrophoresis 2002;23(2):161-6.

17. Wakkee M, Thilo HB, Prens EP, et al. Unfavourable cardiovascular risk profiles in untreated and treated psoriasis patients. Atherosclerosis 2007;190(1):1-9. 
18. Gelfand JM, Neimann AL, Shin DB, et al. Risk of myocardial infarction in patients with psoriasis. JAMA 2006;96(4):1735-41.

19. Poikolainen K, Karvonen J, Pukkala E. Excess mortality related to alcohol and smoking among hospital-treated patients with psoriasis. Arch Dermatol 1999;135(12):1490-3.

20. Henseler T, Christophers E. Disease concomitance in psoriasis. J Am Acad Dermatol 1995;32(6):982-6.

21. Ross R. Atherosclerosis-an inflammatory disease. N Engel J Med 1999;340(2):115-26.

22. Abusamieh M, Ash J. Atherosclerosis and systemic lupus erythematous. Cardiol Rev 2004;12(5):267-75.

23. Speidl WS, Graf S, Hornykewycz S, et al. High- sensitivity $\mathrm{C}$-reactive protein in the prediction of coronary events in patients with premature coronary artery disease. Am Heart J 2002;144(3):449-55.

24. Koenig W. Predicting risk and treatment benefit in atherosclerosis: the role of C-reactive protein. Int J cardiol 2005;98(2):199-206.

25. Vahiquist C. Effects of retinoids on lipoprotein metabolism. Curr Probl Dermatol 1991;20:73-8.

26. Katz HI, Waalen J, Leach EE. Acitretin in psoriasis: an overview of adverse effects. J Am Acad Dermatol 1999;41(3 Pt 2):S7-12.

27. Stiller MJ, Pak GH, Kenny C, et al. Elevation of fasting serum lipids in patients treated with low-dose cyclosporine for serve plaque- type psoriasis. An assessment of clinical significance when viewed as a risk factor for cardiovascular disease. J AM Acad dermatol 1992;27(3):434-8.

28. Hamsten A. Hypertriglyceridaemia, triglyceride-rich lipoproteins and coronary heart disease. Bailliers Clin Endocrinol Metab 1990;4(4):895-922.
29. Kowalska MA, Tuszynski GP, Capuzzi DM. Plasma lipoproteins mediate platelet adhesion. Biochem Biphys Res Commun 1990;172(1):113-8.

30. Austin MA. Triglyceride, small, dense low-density lipoprotein, and the atherogenic lipoprotein phenotype. Curr Atheroscler Rep 2007;2(3):200-7.

31. Dahlen GH, Guyton JR, Attar M, et al. Association of levels of lipoprotein $\mathrm{Lp}(\mathrm{a})$, plasma lipids, and other lipoproteins with coronary artery disease documented by angiography. Circulation 1986;74(4):758-65.

32. Tam LS, Tomlinson B, Chu TT, et al. Cardiovascular risk profile of patients with psoriatic arthritis compared to controls--the role of inflammation. Rheumatology 2008;47(5):718-23.

33. Krueger JG. The immunologic basic for the treatment of psoriasis with new biologic agents. J Am Acad Dermatol 2002;46(1):1-3; quiz 23-6.

34. Prodanowich S, Ma F, Taylor JR, et al. Methotrexate reduced incidence of vascular disease in veterans with psoriasis or rheumatoid arthritis. J Am Acad Dermatol 2005;52(2):262-7.

35. Gelfand JM, Neimann AL, Shin DB, et al. Risk of myocardial infarction in patients with psoriasis. JAMA 2006;296(14):1735-41.

36. Eikelboom JW, Lonn E, Genest J, et al. Homocyst(e)ine and cardiovascular disease: a critical review of the epidemiologic evidence. Ann Inter Med 1999;131(5):36375.

37. McCully KS, Wilson RB. Homocysteine theory of arteriosclerosis. Atherosclerosis 1975;22(2):215-27.

38. Gudionsson JE, Elder JT. Psoriasis. In: Wolff K, Goldsmith LA, Katz SI. (eds) Fitzpatrick's dermatology in general medicine. $7^{\text {th }}$ edn. vol.1. 2008:183-4. 